\title{
New Scheme for Spontaneous Symmetry Breaking of Color SU(3)
}

\author{
A. Schäfer, B. Müller, and W. Greiner \\ Institut für Theoretische Physik der Johann Wolfgang Goethe-Universität, \\ D-6000 Frankfurt am Main 11, Germany \\ (Received 11 January 1983)
}

\begin{abstract}
A new spontaneous-symmetry-breaking mechanism is formulated for $\mathrm{SU}(3)$, which is used to describe the formation of bags around quarks. The Higgs field is replaced by the scalar product of two colored fermion fields. This model gives mass only to one gluon (equivalent to $A_{\mu}{ }^{8}$ ) when spontaneously broken. The consequences of this scheme are discussed, and it is argued that it can explain several puzzling high-energy heavy-ion experiments.
\end{abstract}

PACS numbers: 11.15.Ex, 11.30.Jw, 12.35.Ht, 25.70.Np

Various attempts have been made to describe quark confinement as a mechanism that is analogous to superconductivity. ${ }^{1-4}$ This analogy comes about as follows. The Lagrangian describing superconductivity is invariant under local phase changes of the electron field. However, the ground state, which is a Cooper-pair condensate, is not. This generates an effective-mass term for a photon inside the superconductor. ${ }^{5}$ This parallel is realized either phenomenologically, by introducing spontaneous symmetry breaking (SSB), which is equivalent to the phenomenological description of superconductivity by the Landau-Ginzburg theory; or, on a more fundamental level, by dynamical symmetry breaking (DSB), ${ }^{6-11}$ copying the BCS theory. Both ways to attack the problem have their justifications. Phenomenological models, in general, give more physical insight and need less theoretical assumptions, but they usually suffer from being nonrenormalizable. We propose in this work a model which must be regarded as phenomenological in its present state.

We want to formulate a SSB mechanism which takes into account our interpretation of the Higgs particle as being a bound state of two fermions. This is also the point of view adopted by the technicolor models. ${ }^{12}$ However, in contrast to these models we will use SSB instead of DSB. The easiest way to do this is to replace $\Phi(x)$ by

$$
\Phi(x) \rightarrow \bar{\chi}(x) \chi(x),
$$

where $\chi(x)$ transforms under the fundamental representation of the gauge group. We want to emphasize that our model can be formulated equally well with boson fields instead of fermion fields, as long as they transform under the fundamental representation, i.e., only the color degree of freedom is essential. We also replace $\langle\operatorname{vac}|\Phi(x)| \operatorname{vac}\rangle \neq 0$ by

$$
\langle\operatorname{vac}|\bar{\chi}(x) \chi(x)| \operatorname{vac}\rangle \neq 0 \text {. }
$$

We hope that this crude Ansatz will describe adequately all those features which depend only on the color degree of freedom and are insensitive to the precise internal structure of the "Cooper pair." The assumption of a fermion condensate, i.e., of (2), is the $\mathrm{SU}(3)$ equivalent of chiral symmetry breaking. ${ }^{13}$ As $\Phi(x)$ is a scalar field, the vacuum will be a color scalar even after SSB in contrast to the situation in, e.g., the Glashow-Salam-Weinberg model. We modify the kinetic energy term in the standard Higgs Lagrangian,

$$
L(x)=\left|\partial^{\mu} \Phi(x)\right|^{2}+\mu^{2}|\Phi(x)|^{2}-\lambda|\Phi(x)|^{4},
$$

to introduce a coupling to the gauge fields:

$$
L_{\bar{\chi} \chi}=\left|\bar{\chi}\left(\partial_{\mu}-i g \boldsymbol{T}^{a} A_{\mu}{ }^{a}\right) \chi\right|^{2}+\mu^{2}|\bar{\chi} \chi|^{2}-\lambda|\bar{\chi} \chi|^{4} \text {. }
$$

Adding to Eq. (3) the QCD Lagrangian and a quarkHiggs-coupling term we get our model Lagrangian:

$$
\begin{gathered}
L(x)=:\left\{-\frac{1}{4} A_{\mu \nu}{ }^{a} A_{a}{ }^{\mu \nu}+\sum_{i=u_{0} d, \ldots} \bar{\Psi}_{i}\left(p_{\mu} \gamma^{\mu}+g T^{a} A_{a}{ }^{\mu} \gamma_{\mu}-m_{i}\right) \Psi_{i}+\left|\bar{\chi}\left(\partial_{\mu}-i g T^{a} A_{a}{ }^{\mu}\right) \chi\right|^{2}+\mu^{2}|\bar{\chi} \chi|^{2}-\lambda|\bar{\chi} \chi|^{4}\right. \\
\left.-\sum_{i} f_{i} \bar{\Psi}_{i} \Psi_{i} \bar{\chi} \chi[+L \chi(x)]\right\}:
\end{gathered}
$$

The main idea for the use of SSB is the following. We believe that there exists a complete, renormalizable, canonical field theory showing the phenomenon of dynamical symmetry breaking (DSB). However, there is still no comprehensive theory to describe DSB. Therefore we use the semiclassical 
theory of SSB instead as an effective theory. This mixture of quantum-mechanical and classical ideas shows up in Eq. (5). In the DSB scheme $L \chi(x)$ would be essential because it describes the dynamics of the $\chi$ field. In the SSB scheme (which is a kind of low-energy approximation) it is replaced by the effective Higgs potential, i.e., the term $L \chi(x)$ is neglected (we therefore enclosed it in brackets). $\left|\bar{\chi} g T^{a} A_{a}{ }^{\mu} \chi\right|^{2}$ is an effective second-order interaction term.

To discuss the consequences of (5) we start by explaining how (5) can lead to the creation of bags. What one does in SSB is to minimize the vacuum expectation value (VEV) of the Higgs potential with respect to the physical vacuum $\mid$ vac $\rangle$, as opposed to the pertubative vacuum $|0\rangle$ for which $\langle 0|\Phi| 0\rangle \equiv 0$. This means that in a situation where real quarks are actually present one has to include the additional term

$$
-\left\langle\operatorname{vac}\left|\sum_{i} f_{i} \bar{\Psi}_{i} \Psi_{i} \bar{\chi} \chi\right| \operatorname{vac}\right\rangle \equiv \alpha(x) \rho(x)
$$

in the vacuum energy functional. For most models using SSB the fermions are massless in the beginning. Then the term (6) vanishes because $\bar{\Psi} \Psi \equiv 0$ for massless spinor fields. However, in our model the quarks are initially massive, and therefore the term (6) contributes. Also, the coupling constants $f_{i}$ must be chosen very large in order to give a high mass to a single quark without a surrounding bag in the physical vacuum. When we set $\langle\operatorname{vac}|: \bar{\chi} \chi:| \operatorname{vac}\rangle=\rho$, the $\mathrm{VEV}$ of the Higgs potential reads

$$
V_{\rho}(x)=-\mu^{2} \rho(x)^{2}+\lambda \rho(x)^{4}+\alpha(x) \rho(x) .
$$

For $\alpha(x)>\left(\frac{2}{3}\right)^{3 / 2}\left(\mu^{3} / \sqrt{\lambda}\right)$ there is a single minimum of $V_{\rho}$ at $\rho=0$, and the symmetry remains unbroken. As already indicated in (7) the space dependence of $\alpha(x)$ leads to a space dependence of $\rho(x)$ depicted schematically in Fig. 1. This figure shows a qualitative guess guided by the work of Goldflam and Wilets, and many other works (Ref. 14 and references therein). It shows how quantum-mechanical fluctuations give rise to a transition region with a width denoted by $\tilde{r}$ in Fig. 1 . The difference between $V_{\rho}$ inside and outside of the bag leads to a bag pressure

$$
B \approx \mu^{4} / 4 \lambda \text {. }
$$

The nonvanishing VEV which causes the SSB in our model is (with use of hats to mark operators)

$$
\langle\operatorname{vac}|: \hat{\bar{\chi}} \hat{\chi}:| \operatorname{vac}\rangle=\rho(x) \text {. }
$$

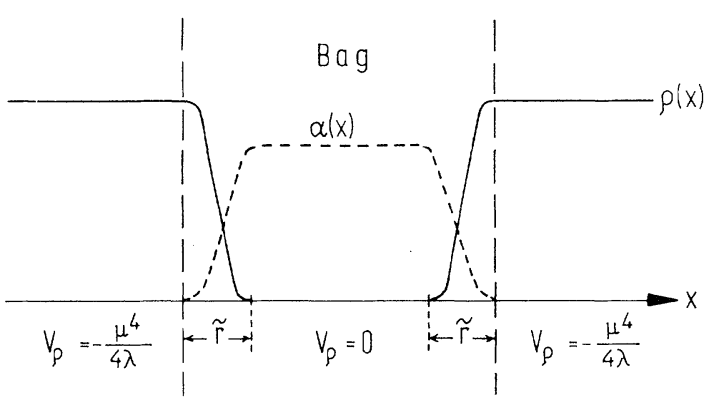

FIG. 1. The creation of quark bags by the prevention of SSB ( $\tilde{r}$ denotes the width of the transition region).

We set

$$
\hat{\chi}=\chi_{0}+\hat{\zeta} \text { with }\langle\operatorname{vac}|: \hat{\bar{\zeta}} \hat{\zeta}:| \operatorname{vac}\rangle=0 \text {. }
$$

$\hat{\chi}$ and $\hat{\zeta}$ differ in the states with respect to which they are normal ordered. We parametrize this difference as follows:

$$
(\hat{\chi}-\hat{\zeta})|\operatorname{vac}\rangle=\chi_{0}|\operatorname{vac}\rangle \text {. }
$$

In the spirit of SSB we set

$$
\hat{\chi}(x)=\chi_{0}(x)+\hat{\zeta}(x)=[\rho(x)]^{1 / 2}\left(\begin{array}{l}
\alpha(x) \\
\beta(x) \\
\gamma(x)
\end{array}\right)+\hat{\zeta}(x),
$$

with a parametrization subject to the condition

$$
\begin{aligned}
& \bar{\alpha}(x) \alpha(x)+\bar{\beta}(x) \beta(x)+\bar{\gamma}(x) \gamma(x)=1, \\
& \rho(x)>0 .
\end{aligned}
$$

In the arbitrariness of $\alpha, \beta$, and $\gamma$, the gauge invariance shows up. With Eq. (11), the gluonmass matrix

$$
M_{a b}^{2}=2\left\langle\operatorname{vac}\left|: g^{2}\left(\hat{\bar{\chi}} T^{a} \hat{\chi}\right)\left(\hat{\bar{\chi}} T^{b} \hat{\chi}\right):\right| \operatorname{vac}\right\rangle
$$

acquires one nonvanishing eigenvalue:

$$
\operatorname{Det}\left(M^{2}-\lambda I\right)=\lambda^{7}\left(\lambda-\frac{2}{3} g^{2} \rho^{2}\right)=0 .
$$

Thus seven gluons remain massless and one gluon acquires the mass $m_{8}=\left(\sqrt{\frac{2}{3}}\right)^{1 / 2} g \rho$. The usual essential ingredient of the Higgs mechanism is that this result does not depend on the choice of $\alpha, \beta$, and $\gamma$ in Eq. (11). Setting $\alpha=\beta=0$ and $\gamma=1$, we see that the massive gluon is gauge equivalent to $A_{8}$. (Only $T^{8}=\frac{1}{2} \lambda_{\mu}{ }^{8}$ has a nonvanishing 3,3 component.)

It has been argued by DeRújula, Giles, and Jaffe $^{11}$ (RGJ) that massive gluons can be unconfined, although they did not give a strict proof of this conjecture. In the following we shall carry over their arguments to our model. However, be- 
fore doing so we want to stress one major difference between the model of RGJ and our work. In Ref. 11 all gluons become massive and deconfined. Hence, if a $q \bar{q}$ pair is pulled apart, no string builds up and the quarks can be separated. In our model solely the $A_{\mu}{ }^{8}$ gluon gets deconfined. Therefore, a string will be formed by the other seven gluons [ which include an unbroken SU(2) subgroup]. Thus quarks are expected to remain confined (Fig. 2). According to Fig. 1 the $A_{\mu}{ }^{8}$ gluons are massive only near the edge of and outside of the bag, $m_{8}(x) \sim \rho(x)$. The equation for the timelike component of the color field is

$$
\left[\nabla^{2}-m_{a}{ }^{2}(x)\right] A_{a}{ }^{0}(x)=-g \rho_{a}(x),
$$

where

$$
m_{a}=m_{8} \delta_{a 8} \text {. }
$$

We are looking for static solutions and have therefore absorbed all nonlinearities in the colorcharge density $\rho_{a}(x)$. Integrating over the bag volume $V$ and using the bag boundary condition for the gluon field leads to

$$
\begin{aligned}
& g \boldsymbol{Q}_{8}=\int_{V} d^{3} x A_{8}^{0}(x) m_{8}^{2}(x) ; \\
& Q_{1}=Q_{2}=\ldots=Q_{7}=0 .
\end{aligned}
$$

Obviously, bags with $Q_{8} \neq 0$ seem to be possible, in particular those containing only one $A_{8}{ }^{\mu}$ gluon. Thus $A_{8}{ }^{\mu}$ gluons are expected to be unconfined. According to Eq. (17), the unbalanced $Q_{8}$ charge is concentrated at the edge of the bag. Let us denote by $\tilde{V}$ that part of the bag volume in which $m_{8}(x) \neq 0$. Then the potential necessary to produce a given charge is inversely proportional to $\tilde{V}$ and $m_{8}^{2}$ :

$$
A_{8}{ }^{0} \sim Q_{8} / m_{8}{ }^{2} \tilde{V}+\ldots \text {. }
$$
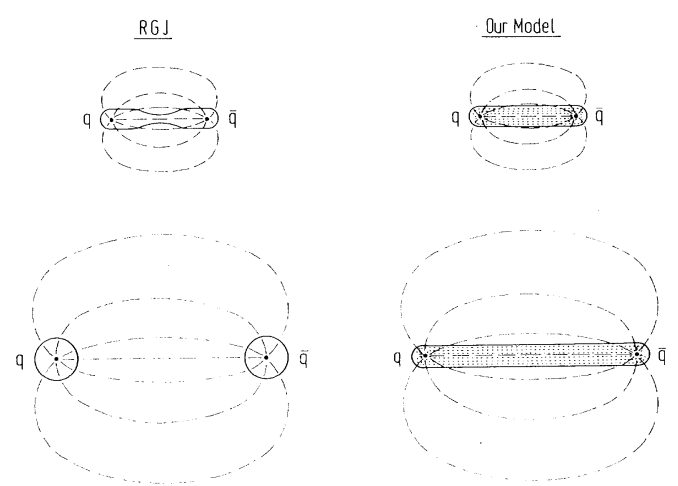

FIG. 2. Quark confinement in our model compared with quark unconfinement in the work of RGJ.
The gluon-mass term the n leads to a bag energy term of the form [ see Eq. (8)]

$$
E=(\text { const }) Q_{8}{ }^{2} / m_{8}{ }^{2} \tilde{V}+B V+\ldots
$$

( $B$ stands for the bag constant, so that $B V$ is the volume energy of the bag). As $\tilde{V}$ will be essentially proportional to the surface of the bag we set $\tilde{V} / V^{2 / 3}=\sigma$. The minimization of $E$ with respect to $V$ leads then to

$$
V \sim\left(Q_{8}^{2} / m_{8}^{2} \sigma B\right)^{3 / 5}+\ldots .
$$

The dots indicate that we have neglected the whole internal dynamics which will show up, for example, as Fermi pressure due to the quarks. Assuming $\sigma^{2} m_{8}$ to be sufficiently small we predict the $Q_{8}$-charged bags to be heavy, large in size, and preferably deformed, as for a bag of given volume $V$ the value of $\sigma$ gets bigger, and thus $E$ smaller, if the bag is deformed. (RGJ assumed $E$ to be of the order of $10 \mathrm{GeV}$.)

Because the volume of the color-charged bag is made large by the gluons there are many unoccupied, low-energy quark states in it. Therefore a color-charged bag can reduce its mass by "swallowing" normal nucleons, gaining about one $B V$ (nucl) per nucleon (i.e., the volume energy per nucleon bag) in the process. Hence such charged bags should be produced most easily in a nuclear surrounding.

Let us notice that there is a severe objection to the mechanism of RGJ put forward by Georgi. ${ }^{15}$ RGJ had to assume that the VEV of the Higgs field is extremely small. However, under this condition quantum-mechanical fluctuations might easily dynamically restore the spontaneously broken symmetry. In our scheme the big mass for colorcharged bags is mainly produced by the smallness of the transition region. Therefore we do not have to assume that the VEV $\rho$ itself is very small.

As argued by RGJ, bags with nonvanishing color charge might be produced by cosmic rays or in experiments at very powerful accelerators. As discussed above it costs less energy to produce a color-charged bag in nuclear surroundings, i.e., to produce a $Q_{8}$-charged bag composed of several hadrons instead of a single one. In addition, in a heavy-ion collision the energy of all the nucleons building this large, color-charged bag could add up collectively. We therefore argue that high-energy heavy-ion collisions are a good place to look for such color-excited states.

In fact, there are several yet unexplained results of heavy-ion experiments which could be 
understood in the light of our model.

First, there is the effect of reduced mean free path ( $\mathrm{mf} p$ ) of secondaries produced in heavy-ion collisions. Friedlander et $a l .{ }^{16}$ claim that they have to assume that a small fraction of the secondary particles produced by a $2-\mathrm{GeV} / \mathrm{u}{ }^{56} \mathrm{Fe}$ beam in nuclear emulsion have drastically enlarged cross sections. These results have been confirmed by an independent group ${ }^{17}$ which reports also an energy threshold between 1 and 2 $\mathrm{GeV} / \mathrm{u}$ for this process. The same effect is also seen for other projectile particles and different energies. ${ }^{16,18,19}$ Finally, a "memory effect" has been reported, ${ }^{16}$ i.e., "anomalous" secondaries have an enhanced probability to produce "anomalous" tertiaries, and so on. A completely different analysi $\mathrm{s}^{19}$ of the same and additional raw data suggests that a certain fraction of all stars produced in the nuclear emulsion corresponds to a temperature raised be a factor of 4 over that of "normal" stars. These experiments can be qualitatively explained if one assumes that in a certain fraction of these high-energy heavy-ion collisions $Q_{8}$-charged bags are produced. These would have an increased cross section leading to the short-mfp effect. Those stars in which the excited states are produced would correspond to the "hot" stars of Ref. 19.

We note that there have been proposed other mechanisms of color excitation to explain the short-mfp effect. These suggestions form two main groups. Either they investigate purely internal excitations ${ }^{20}$ (these models have problems in explaining the long lifetime of the anomalous fragments), or they assume the existence of open color and fractionally charged baryons. ${ }^{21}, 22$ Let us stress once again that our model does not predict open triplet-charge color (i.e., red, green, or blue), but only a special type of color-octet charge unique to SU(3). Furthermore, the quarks are still confined in our model and no fractional charges appear. This is very important in the light of some recent experimental and theoretical work..$^{22}$
${ }^{1}$ N. K. Nielsen and P. Olesen, Nucl. Phys. B143, 485 (1978), and B144, 376 (1978).

${ }^{2}$ S. G. Martinyan and G. K. Savvidy, Nucl. Phys. B156, 1 (1979).

${ }^{3}$ D. A. Kirzhnits, Usp. Fiz. Nauk 125, 169 (1978)

[ Sov. Phys. Usp. 21, 470 (1978)].

${ }^{4}$ C. G. Callan, R. F. Dashen, and D. J. Gross, Phys. Rev. D 19, 1826 (1979); G. Mack, Phys. Lett. 78B, 263 (1978).

${ }^{5} \mathrm{~K}$. Huang, Quarks, Leptons, and Gauge Fields (World Scientific, Singapore, 1982).

${ }^{6}$ Y. Nambu and G. Jona-Lasinio, Phys. Rev. 122, 345

(1961); H. Pagels, Phys. Rev. D 14, 2747 (1976).

${ }^{7}$ W. A. Bardeen, M. S. Chanowitz, S. D. Drell, M. Weinstein, and T. M. Yan, Phys. Rev. D 11, 1094 (1975).

${ }^{8}$ L. Susskind, Phys. Rev. D 20, 2619 (1979).

${ }^{9}$ J. Goldstone, A. Salam, and S. Weinberg, Phys. Rev. 127, 965 (1962).

${ }^{10} \mathrm{~J}$. M. Cornwall and R. E. Norton, Phys. Rev. D $\underline{8}$, 3338 (1973).

${ }^{11}$ A. De Rújula, R. C. Giles, and R. L. Jaffe, Phys. Rev. D 17, 285 (1978).

${ }^{12}$ E. Farhi and L. Susskind, Phys. Rep. 74,277 (1981). ${ }^{13}$ Huang, Ref. 5, p. 264.

${ }^{14}$ R. Goldflam and L. Wilets, Phys. Rev. D $\underline{25}, 1951$ (1982).

${ }^{15}$ H. Georgi, Phys. Rev. D 22,225 (1980); A. De Rúula, R. C. Giles, and R. L. Jaffe, Phys. Rev. D 22, 227 (1980).

${ }^{16}$ E. M. Friedlander, R. W. Gimpel, H. H. Heckmann, Y. J. Karant, B. Judek, and E. Ganssauge, Phys. Rev. Lett. 45, 1084 (1980).

${ }^{17}$ P. L. Jain and G. Das, Phys. Rev. Lett. $\underline{48}, 305$ (1982).

${ }^{18}$ H. B. Barber, P. S. Freier, and C. J. Waddington, Phys. Rev. Lett. $\underline{48}, 856$ (1982); W. Heinrich et al., to be published.

${ }^{19}$ H. G. Baumgardt, E. M. Friedlander, and E. Schopper, J. Phys. G 7, L175 (1981).

${ }^{20}$ S. Fredriksson and M. Jändel, Phys. Rev. Lett. 48, 14 (1982); W. J. Romo and P. J. S. Watson, Phys. Lett. 88B, 354 (1979).

${ }^{21}$ R. Slansky, T. Goldman, and G. L. Shaw, Phys. Rev. Lett. 47, 887 (1981); G. F. Chapline, Phys. Rev. D 25, 911 (1982); R. Saly, M. K. Sundaresan, and P. J. S. Watson, Phys. Lett. 115B, 239 (1982).

${ }^{22}$ G. Lazarides, Q. Shafi, and W. P. Trower, Phys. Rev. Lett. 49, 1756 (1982). 